\title{
INVERTING THE HALF-JUMP
}

BY

\author{
S. HOMER AND G. E. SACKS ${ }^{1}$
}

\begin{abstract}
Assume $\beta$ is weakly admissible over 0 and $0^{1 / 2}$. It follows that the $\beta$-recursively enumerable degrees are dense. In addition each $\beta$-recursively enumerable degree above $0^{1 / 2}$ is the half-jump of some tamely $\beta$-recursively enumerable degree below $0^{1 / 2}$.
\end{abstract}

Introduction. The first jump inversion theorem was found by R. Friedberg [1]. He showed by means of an embryonic forcing argument that the range of the Turing jump is the cone of degrees above $0^{\prime}$. A refinement of his theorem, obtained by an infinite injury argument [2], states that the range of the Turing jump, restricted to the recursively enumerable degrees, is the set of degrees above and recursively enumerable in $0^{\prime}$. A distant descendant of that result is proved below in the setting of $\beta$-recursion theory. The argument draws on most of the ideas in [2]. Difficulties arise not merely because $\omega$ has been supplanted by $\beta$, but more because $\beta$ is not assumed to be $\Sigma_{1}$ admissible.

A density theorem for the $\beta$-recursively enumerable degrees is also proved below for certain inadmissible $\beta$ 's. The work of Maass [3] on regular, $\beta$-recursively enumerable sets in the weakly admissible case is applied extensively.

$\beta$-recursion theory $[4,5]$ is the study of $\Sigma_{1}$ definability on $S_{\beta}$, where $\beta$ is an inadmissible (that is, not $\Sigma_{1}$ admissible) limit ordinal. Such an $S_{\beta}$ is a typical initial segment of the Jensen $J$ hierarchy for $L$. The intent of $\beta$-recursion theory is to adapt familiar ideas of classical recursion theory to rudimentarily closed, but otherwise arbitrary, initial segments of $L$. Throughout this paper it is assumed that $\beta$ is weakly admissible, that is, the $\Sigma_{1}$ cofinality of $\beta(\sigma 1 \mathrm{cf} \beta)$ is at least the $\Sigma_{1}$ projectum of $\beta$ $\left(\beta^{*}\right)$. The work of S. Friedman [5] and W. Maass $[3,6]$ has greatly illuminated the weakly admissible case. They have managed to show us the following.

There is a $\beta$-recursively enumerable degree $0^{1 / 2}$, strictly between 0 and $0^{\prime}$. It is the degree of the complete $\Delta_{1}^{S_{\beta}}$ set. (For this only inadmissibility is needed; now assume weak inadmissibility.) Below $0^{1 / 2}$ there is a subcollection of the $\beta$-r.e. degrees, called tame, that exhibit most of the properties associated with the admissible case.

Above $0^{1 / 2}$ Maass [6] has determined which $\beta$-r.e. degrees have regular, $\beta$-r.e. representatives. Little more is known without the additional assumption that $\beta$ is weakly admissible over $0^{1 / 2}$. In particular it is not known if weak admissibility alone

Received by the editors December 10, 1980, and, in revised form, May 21, 1982.

1980 Mathematics Subject Classification. Primary 03D60; Secondary 03D25, 03E45.

${ }^{1}$ The second author was supported in part by NSF grant number MCS78-07344. Several comments by W. Maass were most helpful. 
implies the existence of a $\beta$-r.e. degree strictly between $0^{1 / 2}$ and $0^{\prime}$. The additional assumption does yield density, as will be seen below, and also that each $\beta$-r.e. degree above $0^{1 / 2}$ is the half-jump of a tamely $\beta$-r.e. degree. These results will be applied to $\alpha$-recursion theory to obtain information about degrees above $0^{\prime}$. Such an application is to be expected since the adjunction of $0^{\prime}$, the complete $\alpha$-r.e. set, to $L_{\alpha}$ often yields a weakly admissible structure.

Our results can be obtained in either of two ways. The first is direct: arguments from $\alpha$-recursion theory are modified so as to allow weak admissibility to do all the work; the main difficulty to be circumvented is the presence of $\alpha$-finite sets that take forever, more precisely the entire life of the construction, to enumerate. The second, originated by Maass [3], is to replace $S_{\beta}$ by an admissible structure, the so-called admissible collapse. The second approach is cleaner than the first, but its details are elusive. Both are utilized below. It seems likely that the first approach must be pursued if further progress is to be made in the face of ever increasing inadmissibility and nonregularity.

$\S 2$ contains background, essential definitions, and preliminary observations. $\S 3$ is devoted to the inversion of the half-jump by the direct method. Two corollaries are given in $\$ 4$. A density theorem is proved in $\$ 5$ via the indirect method, and some open problems are stated in the final section.

2. Machinery. The fundamentals of $\beta$-recursion theory were introduced in [4]. For details see [5]. The idea is to do recursion theory on an arbitrary rudimentarily closed initial segment of $L$ with r.e. taken to be $\Sigma_{1}$. The sole departure from $\alpha$-recursion theory is the dropping of $\Sigma_{1}$ admissibility. The Jensen $S$ hierarchy for $L$ is ideal for our purposes. Suppose $\beta$ is a limit ordinal and $A \subseteq S_{\beta}$. $A$ is said to be $\beta$-recursively enumerable ( $\beta$-r.e.) if $A$ has a $\Sigma_{1}$ definition over $S_{\beta}$ with parameters in $S_{\beta} . A$ is $\beta$-recursive ( $\beta$-rec.) if both $A$ and $S_{\beta}-A$ are $\beta$-r.e. $A$ is $\beta$-finite if $A \in S_{\beta} . A$ is regular if $A \cap x$ is $\beta$-finite for every $\beta$-finite $x$.

It will on occasion be necessary to approximate $\beta$-recursive functions by $\beta$-finite functions as follows. Let $f: S_{\beta} \rightarrow S_{\beta}$ be $\beta$-recursive. Suppose $(E z) R(x, y, z)$ is $\Sigma_{1}$ over $S_{\beta}$ and defines the graph of $f$. Define

$$
f_{\sigma}=\left\{\langle x, y\rangle \mid S_{\gamma} \vDash(E z) R(x, y, z)\right\}
$$

for each $\sigma \in S_{\beta}$, where $\gamma$ is the least $\delta$ such that $\sigma \in S_{\delta}$.

The standard complete $\beta$-r.e. set $C$ is $\left\{\langle e, x\rangle \mid S_{\beta} \vDash \phi_{e}(x)\right\}$, where $\left\{\phi_{e}(x) \mid e \in S_{\beta}\right\}$ is a $\beta$-recursive enumeration of all $\Sigma_{1}^{\beta}$ formulas with free variable $x$.

Relative $\beta$-recursiveness is defined as in $\alpha$-recursion theory. A reduction procedure $\{e\}$ is a $\beta$-r.e. collection of quadruples $\langle H, J, x, y\rangle$ of elements of $S_{\beta}$.

$$
\{e\}^{A}(x)=y \leftrightarrow(E H)(E J)\left[\langle H, J, x, y\rangle \in\{e\} \& H \subseteq A \& J \subseteq S_{\beta}-A\right] .
$$

Note that $\{e\}$ need not be single valued. $\{e\}_{\sigma}$ is an approximation of $\{e\}$ corresponding to that part of $\{e\}$ enumerated prior to stage $\sigma$ of the universal enumeration.

$f$ is weakly $\beta$-recursive in $A\left(f \leqslant_{w \beta} A\right)$ if $f=\{e\}^{A}$ for some $e \in S_{\beta} . B$ is $\beta$-recursive in $A\left(B \leqslant_{\beta} A\right)$ if, for some $e$ and all $z \in S_{\beta}$,

$$
z \subseteq B \leftrightarrow\{e\}^{A}(z)=0, \quad z \subseteq \bar{B} \leftrightarrow\{e\}^{A}(z)=1 .
$$

$A$ has the same $\beta$-degree as $B\left(A \equiv_{\beta} B\right)$ if $A \leqslant_{\beta} B$ and $B \leqslant_{\beta} A$. 
Warning. $\beta$-recursive sets often fail to be $\beta$-recursive in the empty set.

A $\beta$-r.e. set is said to be tamely $\beta$-r.e. if there is some $\beta$-r.e. enumeration $\left\{A^{\sigma} \mid \sigma<\beta\right\}$ of $A$ such that if $K \subseteq A$ and $K$ is $\beta$-finite, then $K \subseteq A^{\sigma}$ for some $\sigma$. Of course if $\beta$ is $\Sigma_{1}$ admissible, then every $\beta$-r.e. set is tamely $\beta$-r.e.

Various definable singularities are needed to do recursion theory on inadmissible $S_{\beta}$ 's. The $\Sigma_{n}^{\beta}$ cofinality of $\beta(\sigma n \operatorname{cf}(\beta))$ is the least $\gamma \leqslant \beta$ such that some $\Sigma_{n}^{\beta} f$ maps $\gamma$ unboundedly into $\beta$. The $\Sigma_{n}^{\beta}$ projectum of $\beta(\sigma \mathrm{np}(\beta))$ is the least $\gamma \leqslant \beta$ such that there exists a one-one $\Sigma_{n}^{\beta} f$ that maps $S_{\beta}$ into $\gamma . \beta^{*}$ denotes $\sigma \operatorname{lp}(\beta)$, and $\kappa$ denotes $\sigma \mathrm{lcf}(\beta)$.

In accord with Maass [6], $\beta$ is weakly inadmissible if $\beta>\kappa \geqslant \beta^{*}$. For the remainder of this paper, $\beta$ is weakly inadmissible ordinal.

Proposition 2.1 (S. Friedman [5]). There exists a one-one, $\beta$-recursive map of $S_{\beta}$ onto $\kappa$.

For each $A \subseteq S_{\beta}$, define $\kappa^{A}$ to be

$$
\mu \gamma_{\gamma \leqslant \kappa} \leqslant(E f)\left[f \leqslant_{w \beta} A \& f: \gamma \stackrel{\text { unboundedly }}{\rightarrow} \kappa\right] .
$$

$\kappa^{A}$ is the recursive cofinality of $A$. In the next section a nontrivial $A$ will be constructed so that $\kappa^{A}=\kappa$. The next proposition will be needed for that end.

Proposition 2.2. If $\kappa^{A}<\kappa$, then $\kappa^{A} \leqslant \beta^{*}$.

Proof. Suppose not. Then $\beta^{*}<\kappa^{A}<\kappa$. Let

$$
f: S_{\beta} \stackrel{1-1}{\rightarrow} \beta^{*} \text { and } g: \kappa^{A} \rightarrow \beta
$$

where $f$ is $\beta$-recursive, $g \leqslant_{w \beta} A$ and range $g$ is unbounded in $\beta$. Since $\kappa^{A}<\kappa, f \uparrow \kappa^{A}$ is $\beta$-finite, and so $V=f\left[\kappa^{A}\right]$ is $\beta$-finite. Then $g f^{-1}: V \rightarrow \beta$ is weakly $\beta$-recursive in $A$, has domain whose cardinality (in $S_{\beta}$ ) is less than $\kappa^{A}$, and has range unbounded in $\beta$. A contradiction.

The $\beta$-cardinals are those ordinals less than $\beta$ that are cardinals in the sense of $S_{\beta}$.

Proposition 2.3. If $\beta^{*}$ is a singular $\beta$-cardinal and $\kappa^{A}<\kappa$, then $\kappa^{A}<\beta^{*}$.

Proof. By $2.2, \kappa^{A} \leqslant \beta^{*}$. For a contradiction assume $\kappa^{A}=\beta^{*}$. Since $\beta^{*}$ is singular, there exist a $\delta<\beta^{*}$ and a $\beta$-finite $f: \delta \rightarrow \beta^{*}$ with the range of $f$ cofinal in $\beta^{*}$. Let $g: \kappa^{A} \rightarrow \beta$ be as in the proof of Proposition 2.2. In addition let $g$ be monotonic. Then $g f: \delta \rightarrow \beta$ is weakly $\beta$-recursive in $A$ and has range unbounded in $\beta$, a contradiction.

Proposition 2.4. Let $\lambda<\kappa$ be a regular $\beta$-cardinal, $\gamma<\lambda$, and $\left\{V_{e} \mid e<\gamma\right\}$ a $\beta$-recursively enumerable sequence of $\beta$-finite sets, each of $\beta$-cardinality less than $\lambda$. Then $\cup\left\{V_{e} \mid e<\gamma\right\}$ is $\beta$-finite and has $\beta$-cardinality less than $\lambda$.

Proof. As in Lemma 2.3 of [7]. The assumption of $\Sigma_{1}$ admissibility has been replaced by $\lambda<\kappa$. 
The half-jump was originated by S. Friedman [5]. Let $A \subseteq S_{\beta}$. Fix $g: \kappa^{A} \rightarrow \beta$ so that $g$ has unbounded range and is weakly $\beta$-recursive in $A$. Then $A^{1 / 2}$, the half-jump of $A$, is

$$
\left\{\langle e, z, \sigma\rangle\left|S_{g(\sigma)}\right|=\{e\}^{A}(z) \text { is defined }\right\} .
$$

Proposition 2.5 (S. Friedman [5]).

(i) $A^{1 / 2} \leqslant_{w \beta} A$.

(ii) $A^{\prime} \leqslant_{w \beta} A^{1 / 2}$, where $A^{\prime}$, the jump of $A$, is $\left\{\langle e, z\rangle \mid\{e\}^{A}(z)\right.$ is defined $\}$.

(iii) $(B)\left[A^{\prime} \leqslant_{w \beta} B \rightarrow A^{1 / 2} \leqslant_{\beta} B\right]$.

(iv) If $B$ is $\Pi_{1}$ over $\left\langle S_{\beta}, A\right\rangle$, then the predicate $x \in S_{\beta} \& x \subseteq B$ is weakly $\beta$ recursive in $A^{1 / 2}$.

Proof. To verify (iii) let $e_{0}$ and $e_{1}$ be indices such that

$$
z \in A^{1 / 2} \leftrightarrow\left\{e_{0}\right\}^{A}(z) \text { diverges, } \quad z \notin A^{1 / 2} \leftrightarrow\left\{e_{1}\right\}^{A}(z) \text { diverges. }
$$

Let $f$ be a $\beta$-recursive function such that for all $e$ and $F \in S_{\beta}$,

$$
\{f(e)\}^{A}(F) \text { diverges } \leftrightarrow(z)\left[z \in F \rightarrow\{e\}^{A}(z) \text { diverges }\right] \text {. }
$$

Then

$$
F \subseteq A^{1 / 2} \leftrightarrow\left\{f\left(e_{0}\right)\right\}^{A}(F) \text { diverges, } \quad F \subseteq \overline{A^{1 / 2}} \leftrightarrow\left\{f\left(e_{1}\right)\right\}^{A}(F) \text { diverges. }
$$

Thus a $\beta$-finite question about $A^{1 / 2}$ is answered via a single question about $A^{\prime}$. So if $A^{\prime} \leqslant_{w \beta} B$, then $A^{1 / 2} \leqslant_{\beta} B$.

The work of Maass $[3,6]$ on the admissible collapse is needed in $\$ 5$ for the proof of the density theorem. It was his idea to derive results about $\beta$-r.e. degrees from the degree theory of the admissible collapse of $S_{\beta}$.

A set $A \subseteq \kappa$ is $\beta$-bi-immune if $\left(F \subseteq A\right.$ or $F \subseteq \bar{A}$ ) $\rightarrow F \in S_{\kappa}$ for all $F \in S_{\beta}$. (Recall that $\kappa=\sigma 1 \operatorname{cf}(\beta)$.) Observe that $\beta$-bi-immunity implies tameness for $\beta$-r.e. sets.

Proposition 2.6 (MaAss [3]). There exists a $\beta$-recursive partition $\{A, B, T\}$ of $\kappa$ such that for $X \subseteq \kappa$, if $A \subseteq X$ and $B \subseteq \bar{X}$, then $X$ is $\beta$-bi-immune. In addition $T$ has ordertype $\kappa$.

The above is useful for creating $\beta$-b1-immunity. Suppose $Y \subseteq \kappa$ is $\beta$-r.e. (or $\beta$-rec.). Construct $X$ by injecting $Y$ into $T$; that is, the $\gamma$ th element of $T$ is put into $X$ iff $\gamma \in Y$. Then $X$ is bi-immune and $\beta$-r.e. (or $\beta$-rec.).

Bi-immunity is the link between $S_{\beta}$ and its admissible collapse, soon to be defined. The next proposition singles out, as will be seen, the $\beta$-r.e. degrees associated with the collapse.

Proposition 2.7 (MaAss [3]). Let $b$ be a $\beta$-degree. (i), (ii) and (iii) are equivalent.

(i) $b$ has a bi-immune, $\beta$-r.e. representative.

(ii) $b$ has a tamely $\beta$-r.e. representative.

(iii) $b$ has a $\beta$-rec. representative.

Now for the collapse. Let $p: S_{\beta} \rightarrow \kappa$ be $\beta$-recursive, 1-1 and onto, as in 2.1. Define $T \subseteq \beta^{3}$ by

$$
\langle\gamma, e, z\rangle \in T \leftrightarrow S_{\gamma} \vDash \phi_{e}(z) \text { is defined, }
$$


where $\phi_{e}(e<\beta)$ is the $e$ th $\beta$-rec. function. Let $\hat{T} \subseteq \kappa$ be the projection of $T$ :

$$
\hat{T}=\{\langle p \gamma, p e, p z\rangle \mid\langle\gamma, e, z\rangle \in T\} .
$$

The structure $\mathscr{Q}=\left\langle S_{\kappa}, \hat{T}\right\rangle$ is called the admissible collapse of $S_{\beta}$ and is an invention of Maass.

Proposition 2.8 (MaAss [3]). Q is an admissible structure. For any $A \subseteq \kappa, A$ is $\Sigma_{1}^{Q}$ iff $A$ is $\beta$-r.e.

Define $\leqslant_{\mathcal{Q}}$ in parallel with $\leqslant_{\beta}$. The reduction procedures associated with $\leqslant_{\mathcal{Q}}$ are $\Sigma_{1}^{Q}$ sets of 4-tuples. Part of the proof of 2.8 consists of showing that $\hat{T}$ is a regular subset of $S_{\kappa}$. Consequently $Q$-finite has the same meaning as $\kappa$-finite. In addition, any $\beta$-finite set bounded below $\kappa$ is $\kappa$-finite. If $A$ and $B$ are $\beta$-bi-immune subsets of $\kappa$, then

$$
A \leqslant_{Q} B \text { iff } A \leqslant_{\beta} B .
$$

Proposition 2.9 (MAASs [6]). Let $b$ be a $\beta$-degree. (i), (ii) and (iii) are equivalent.

(i) $b$ has a regular $\beta$-r.e. representative.

(ii) $b$ has a $\beta$-r.e. representative, and either $\sigma 2 \operatorname{cf}(\beta) \geqslant \sigma 2 \mathrm{p}(\beta)$ or $0^{1 / 2} \Varangle_{\beta} b$.

(iii) $b$ has a $\beta$-bi-immune representative $A$ that is regular over $S_{\kappa}$, and there is a $\Sigma_{1}^{Q}$ set $B$ such that $B \leqslant_{\mathbb{Q}} A$ and $A$ is tamely $\Sigma_{1}^{\langle Q, B\rangle}$.

The content of (iii) is: $b$ is tamely r.e. over some lesser degree in the admissible collapse of $S_{\beta} .2 .9$ says all regularity (for $\beta$-r.e. sets) owes its existence to tameness over tamely $\beta$-r.e. sets. (See the discussion at the beginning of $\S 5$.)

To complete this section, some results associated with Shore blocking are sketched. A function $f: \gamma \rightarrow \eta$ is said to be tame $\Delta_{2}$ if there exists a $\beta$-recursive function $g$ : $\gamma \times \beta \rightarrow \eta$ such that

(1) $(\delta)_{\delta<\gamma}(E \sigma)(\tau)_{\tau \geqslant \sigma}[f(\delta)=g(\delta, \tau)]$, and

(2) $(\delta)_{\delta<\gamma}(E \sigma)(\alpha)_{\alpha \leqslant \delta}(\tau)_{\tau \geqslant \sigma}[g(\alpha, \tau)=g(\alpha, \sigma)]$.

For each $\eta \leqslant \beta$, the tame $\Delta_{2}$ cofinality of $\eta(\mathrm{t} \delta 2 \operatorname{cf}(\eta))$ is the least $\gamma \leqslant \eta$ such that there is a tame $\Delta_{2} f: \gamma \rightarrow \eta$ whose range is cofinal in $\eta$.

Proposition 2.10 (Friedman [5]). If $\beta$ is weakly admissible, then $\mathrm{t} \delta 2 \operatorname{cf}(\beta)=$ t $\delta 2 \operatorname{cf}\left(\beta^{*}\right)$.

As in Simpson [8] there exist a tame $\Delta_{2}$ function $G_{0}: \operatorname{tg} \delta 2 \operatorname{cf}(\beta) \rightarrow \beta^{*}$ and a $\beta$-rec. approximation $G: \operatorname{t~} \delta 2 \operatorname{cf}(\beta) \times \beta \rightarrow \beta^{*}$ of $G_{0}$ such that:

(i) range of $G_{0}$ is cofinal in $\beta^{*}$.

(ii) $0=G_{0}(0)$ and $G_{0}$ is nondecreasing.

(iii) $0=G(0, \sigma)$ and $\lambda x \mid G(x, \sigma)$ is nondecreasing.

In what follows it will prove helpful to assume that (i)-(iii) hold when appropriate.

3. Inverting the half-jump. Let $A \subseteq S_{\beta}$. $\beta$ is said to be weakly admissible over $A$ if $\sigma \operatorname{lcf}_{A}(\beta) \geqslant \sigma \operatorname{lp}_{A}(\beta)$. The subscript $A$ means that functions $\Sigma_{1}^{\beta}$ in $A$ are to be employed. (If $A$ is regular, then $\Sigma_{1}^{\beta}$ in $A$ is equivalent to weakly $\beta$-recursive in $A$.) Thus $\beta$ is weakly admissible iff $\beta$ is weakly admissible in 0 . It is easily checked (with the assumption that $\beta$ is not $\Sigma_{1}$ admissible) that $\beta$ is weakly admissible over $0^{1 / 2}$ iff $\sigma 2 \operatorname{cf}(\beta) \geqslant \sigma 2 \mathrm{p}(\beta)$. 
The present section is devoted to proving the following inversion theorem.

Theorem 3.1. Suppose $Y$ is $\beta$-r.e., regular and $0^{1 / 2} \leqslant_{\beta} Y$. Then there exists a tamely $\beta$-r.e. $X$ such that $X<_{\beta} 0^{1 / 2}, X^{1 / 2} \equiv_{\beta} Y$ and $\kappa^{X}=\kappa$.

It follows from Proposition 2.9 that a regular, $\beta$-r.e. $Y>_{\beta} 0^{1 / 2}$ exists iff $\sigma 2 \operatorname{cf}(\beta)$ $\geqslant \sigma 2 \mathrm{p}(\beta)$. Thus Theorem 3.1 has a hidden assumption, namely that $\beta$ is weakly admissible over $0^{1 / 2}$ (in addition to the overriding assumption that $\beta$ is weakly inadmissible).

Intuitive account of 3.1. $X$ is going to be a $\beta$-bi-immune subset of $\kappa$, and hence tamely $\beta$-r.e. The method of 2.6 is applied. 2.6 provides pairwise disjoint $\beta$-recursive sets $A, B$ and $T$ whose union is $\kappa$, and which are such that for any $C \subseteq \kappa$, if $A \subseteq C$ and $B \subseteq \bar{C}$, then $C$ is $\beta$-bi-immune. $X$ will be such a $C$.

To make $Y \leqslant_{\beta} X^{1 / 2}$, each $e \in S_{\beta}$ is associated with some $\beta$-recursive subset of $T$ of ordertype $\kappa$. Let $p: S_{\beta} \rightarrow \kappa$ be 1-1, onto and $\beta$-recursive. Since $\kappa$ is a regular $\beta$-cardinal, there exist pairwise disjoint sets $T_{\gamma}(\gamma<\kappa)$, simultaneously $\beta$-recursive, each of ordertype $\kappa$. Then $e \in S_{\beta}$ is associated with $T_{p(e)}$. As elements of $e \cap Y$ are enumerated, corresponding elements of $T_{p(e)}$ are to be planted in $X$. There will be obstacles to the planting, but if all goes well it will turn out that $e \subseteq \gamma$ if all but a bounded (below $\kappa$ ) amount of $T_{p(e)}$ is contained in $X$. This will suffice to show $Y<X^{1 / 2}$.

To obtain $X^{1 / 2} \leqslant_{\beta} Y$, it is enough by Proposition 2.5 to make $X^{\prime} \leqslant_{w \beta} Y$. If during the enumeration of $X$ it appears that $z \in X^{\prime}$, then a commitment is made to preserve that appearance. To be precise, if at stage $\sigma$, the computation of $\{z\}_{\sigma}^{X^{\sigma}}(z)$ converges, then the relevant negative facts about $X^{\sigma}$ are committed to preservation. This strategy makes possible the weak $\beta$-recursiveness of $X^{\prime}$ in $Y, 0^{1 / 2}$.

The tame $\beta$-r.e.-ness of $X$ implies $X \leqslant_{\beta} 0^{1 / 2}$ according to Friedman [5]. Some further preservations are made to insure $0^{1 / 2} \neq_{\beta} X$. The argument requires that $0^{1 / 2}$ be replaced by a $\beta$-bi-immune $\beta$-r.e. subset of $\kappa$ of the same $\beta$-degree as $0^{1 / 2}$. The replacement is legal by Proposition 2.7. Initial segments of equalities of the form $0^{1 / 2}(z)=\{e\}^{X}(z)$ are preserved. If for some $e$, the initial segment preserved had length $\kappa$, then it would follow, that $0^{1 / 2} \leqslant_{\beta} 0$, a contradiction. Thus either an inequality or a divergence must occur.

Holding $\kappa^{X}$ up to $\kappa$ is managed by procedures similar to those found in hyperregularity constructions in $\alpha$-recursion theory. According to Proposition 2.2, if $\kappa^{X}<\kappa$, then $\kappa^{X} \leqslant \beta^{*}$. Hence it suffices to preserve $\{e\}^{X}$ on initial segments of $\beta^{*}$. There is a difficulty, ignored here, but detailed below, if $\beta^{*}$ equals $\kappa$ or is a singular $\beta$-cardinal. If $\{e\}^{X}$ is total on $\kappa^{X}$ and $\kappa^{X}<\kappa$, then the preservation scheme insures $\{e\}^{X}$ restricted to $\kappa^{X}$ is $\beta$-finite, hence bounded below $\beta$.

Superimposed on all of the above plantings and preservations is a Shore blocking argument. Requirements are indexed by ordinals less than $\beta^{*}$, which is divided into $\sigma 2 \operatorname{cf}(\beta)$ blocks, each block of $\beta$-cardinality less than $\beta^{*}$. Within each block there are no conflicts between planting and preservation requirements. All requirements in a given block have the same priority. Those in the first block have top priority.

Formal construction. According to Proposition $2.10, \mathrm{t} \delta 2 \operatorname{cf}(\beta)=\mathrm{t} \delta 2 \operatorname{cf}\left(\beta^{*}\right)$. Let this common value be $\alpha$. Let $G_{0}$ and $G$ be functions as in the last paragraph of $\S 2$. 
Let $f: S_{\beta} \rightarrow \beta^{*}$ be $1-1$ into and $\beta$-rec., and recall that $p: S_{\beta} \rightarrow \kappa$ is $1-1$, onto and $\beta$-rec. Finally let $V: S_{\beta} \rightarrow \alpha$ be a $\beta$-rec. function such that for each $\gamma<\alpha, V^{-1}(\{\gamma\})$ is unbounded in $S_{\beta}$.

The source of $X$ 's bi-immunity is the $A$ of 2.6. Let $g: S_{\beta} \rightarrow \kappa$ be a $1-1 \beta$-rec. function that enumerates $A$.

$X^{\sigma}$ is that part of $X$ enumerated prior to stage $\sigma$. Let $H$ be a set of $\beta$-degree $0^{1 / 2}$ such that $H \subseteq \kappa, H$ is $\beta$-r.e. and $H$ is $\beta$-bi-immune. $\left\{H^{\sigma}\right\}$ is an enumeration of $H$, and $\left\{Y^{\sigma}\right\}$ is an enumeration of $Y$.

Stage $0 . X^{0}=\varnothing$.

Stage $\sigma$. Let $\gamma=V(\sigma)<\alpha$.

Positive requirements. For each $e<G(\gamma, \sigma)$, check if $e \in$ range $f_{\sigma}$. ( $f_{\sigma}$ is that portion of the graph of $f$ enumerated prior to stage $\sigma$.) If so, define $e^{\prime}$ by $f_{\sigma}\left(e^{\prime}\right)=e$. Recall that the sets $T_{\gamma}(\gamma<\kappa)$ are pairwise disjoint, simultaneously $\beta$-rec. sets, each of ordertype $\kappa$. A positive requirement $Q\left(\gamma, p\left(e^{\prime}\right), \delta\right)$ for the $\delta$ th element of $T_{\left\langle p\left(e^{\prime}\right), \gamma\right\rangle}$ is created if:

(i) $\delta$ is the least ordinal $\tau$ for which there is no positive requirement for the $\tau$ th element of $T_{\left\langle p\left(e^{\prime}\right), \gamma\right\rangle}$,

(ii) $\delta \subseteq$ range $p_{\sigma}$, and

(iii) $p_{\sigma}^{-1}[\delta] \cap e^{\prime} \subseteq Y^{\sigma}$.

Negative requirements. Three types may be created at stage $\sigma$.

A negative $C$-requirement $N_{\gamma, e}^{C}$ of priority $\gamma$ is created at stage $\sigma$ if:

(i) $e<G(\gamma, \sigma)$,

(ii) $e \in$ range $f_{\sigma}$,

(iii) $\left\{f_{\sigma}^{-1}(e)\right\}_{\sigma}^{X^{\sigma}}\left(f_{\sigma}^{-1}(e)\right)$ is defined, and

(iv) there is no current requirement $N_{\gamma, e}^{C}$.

Let $\delta$ be the supremum of all ordinals that figure negatively in the computation of (iii). Define $N_{\gamma, e}^{C}$ to be $\overline{X^{\sigma}} \cap \delta$. Thus $N_{\gamma, e}^{C}$ serves to preserve a value of $X^{\prime}$, namely $f_{\sigma}^{-1}(e) \in X^{\prime}$. If an element of $N_{\gamma, e}^{C}$ is put in $X$ at some future stage, then $N_{\gamma, e}^{C}$ is said to be destroyed at that stage (and good riddance).

The notion of negative requirement $N_{\gamma, e, z}^{L}$ requires the following definition. A reduction procedure $\{e\}$ is said to be $\gamma$-active at stage $\sigma$ unless there is a current negative requirement $N_{\gamma, e, z^{\prime}}^{L}$ with associated computation

$$
\left\{f_{\tau}^{-1}(e)\right\}_{\tau}^{X^{\tau}}\left(z^{\prime}\right)=H^{\tau}\left(z^{\prime}\right)
$$

such that $H^{\tau}\left(z^{\prime}\right) \neq H^{\sigma}\left(z^{\prime}\right)$. Otherwise $e$ is $\gamma$-inactive.

A negative $L$-requirement $N_{\gamma, e, z}^{L}$ of priority $\gamma$ is created at stage $\sigma$ if:

(i) $z$ is the least $\nu$ such that there is no current $L$-requirement $N_{\gamma, e, \nu}^{L}$,

(ii) $e<G(\gamma, \sigma)$ is $\gamma$-active,

(iii) $f_{\sigma}^{-1}(e)$ is defined, and

(iv) $\left\{f_{\sigma}^{-1}(e)\right\}_{\sigma}^{X^{o}}(z)=H^{\sigma}(z)$.

Let $\delta$ be the supremum of all ordinals that figure negatively in the computation of (iv). Define $N_{\gamma, e, z}^{L}=\overline{X^{\sigma}} \cap \delta$. Thus $L$-requirements preserve equalities between $\left\{f^{-1}(e)\right\}^{X}$ and $H\left(\equiv_{\beta} 0^{1 / 2}\right)$ in order to obtain $0^{1 / 2} \$_{\beta} X$. If, at some later stage, requirement $N_{\gamma, e, z}^{L}$ is destroyed, all requirements $N_{\gamma, e, z^{\prime}}^{L}$ with $z^{\prime}>z$ are also destroyed. This ensures that the equality is preserved on initial segments of $\kappa$. If $e$ is 
$\gamma$-inactive, then some equality committed to preservation earlier has become an inequality only because the $H$ approximation has changed. In that event there is a witness to the inequality of $\left\{f^{-1}(e)\right\}^{X}$ and $H$, and so there is no immediate need to establish mere $L$-requirements on $e$.

$B$-requirements fall into two cases. First suppose either $\beta^{*}=\kappa$ or $\beta^{*}$ is a singular $\beta$-cardinal. For each $e<G(\gamma, \sigma)$, let $z$ be the least $y<e$ for which there is no current $B$-requirement $N_{\gamma, e, y}^{B}$ at stage $\sigma$. A negative $B$-requirement $N_{\gamma, e, z}^{B}$ of priority $\gamma$ is created at stage $\sigma$ if:

(i) $e \in$ range $f_{\sigma}$, and

(ii) $\left\{f_{\sigma}^{-1}(e)\right\}_{\sigma}^{X^{\sigma}}(z)$ is defined.

Let $\delta$ be the supremum of all ordinals that figure negatively in the computation of (ii). Define $N_{\gamma, e, z}^{B}$ to be $\delta \cap \overline{X^{\sigma}}$. If an element of $N_{\gamma, e, z}^{B}$ is put into $X$ at some future stage, then of course $N_{\gamma, e, z}^{B}$ is destroyed, but in addition $N_{\gamma, e, z^{\prime}}^{B}$ is destroyed for all $z^{\prime}>z$. Thus initial segments of $\left\{f^{-1}(e)\right\}^{X}$ are preserved in order to insure $\kappa^{X}=\kappa$.

Now suppose $\beta^{*}<\kappa$ and $\beta^{*}$ is a regular cardinal. The sole change above is to replace " $y<e$ " in the definition of $z$ by " $y<\beta^{*}$ ".

The first step at stage $\sigma$ consists of computing $g(\sigma)$ and putting it into $X$. The second step is devoted to the creation of positive and negative requirements as described above. The third step has $\alpha$ successive substeps. (Recall $\alpha$ is $\mathrm{t} \delta 2 \operatorname{cf}(\beta)$ ) It consists of working through all priorities $\gamma<\alpha$, beginning with $\gamma=0$, and putting elements associated with current positive requirements of priority $\gamma$ into $X$ unless they are restrained by some negative requirement of priority $\gamma^{\prime}<\gamma$. Keep in mind that an element put into $X$ for the sake of positive priority $\gamma$ may destroy some negative requirement of priority $\gamma^{\prime \prime} \geqslant \gamma$ and thereby allow the addition of some other element for the sake of positive priority $\gamma^{\prime \prime \prime}>\gamma^{\prime \prime}$.

Positive requirement $\gamma_{1}$ is rightfully said to have higher priority than negative requirement $\gamma_{2}$ if $\gamma_{1} \leqslant \gamma_{2}$.

The above account of steps at stage $\sigma$ is most apt if $\sigma$ is a successor ordinal. If $\sigma$ is a limit, then the third step of the above procedure is carried out as a preliminary step. The three steps at stage $\sigma$ are then done in their usual order. In this manner it is guaranteed that at the beginning of every stage, every element in a positive requirement is also in $X$ unless blocked by a current negative requirement of higher priority.

Now a sequence of lemmas is proved whose import is that all requirements are met by the above construction. First, several useful distinctions. A negative requirement is said to be permanent if it is never destroyed, otherwise it is temporary. If temporary, it is current prior to its destruction. If permanent, then its associated computation is preserved throughout the construction of $X$ and so is an actual computation relative to $X$.

Second, note that the $\beta$-bi-immunity of $X$, assured by putting $g(\sigma)$ into $X$, implies that each permanent negative requirement is a set of $\beta$-cardinality less than $\kappa$. Since $X$ is $\beta$-r.e., its $\beta$-bi-immunity also implies it is tamely $\beta$-r.e.

Lemma 3.2. For each $\gamma<\alpha\left(=\mathrm{t} \delta 2 \operatorname{cf}\left(\beta^{*}\right)\right)$, the collection of all $z<\kappa$ in positive requirements of priority $<\gamma$ is $\beta$-recursive. 
Proof. Fix $\gamma<\alpha$. $G_{0}(\gamma)<\beta^{*} \leqslant \kappa$, so $\cup f^{-1}\left[G_{0}(\gamma) \cap\right.$ range $\left.f\right]$ is contained in some $S_{\delta_{0}}$, where $\delta_{0}<\beta$. Since $Y$ is regular, $Y \cap S_{\delta_{0}}$ is $\beta$-finite. Now $z$ lies in some positive requirement with priority $<\gamma$ if $z$ satisfies the following $\Delta_{1}^{\beta}$ condition: $z$ is the $\eta$ th element of some $T_{\left\langle p f^{-1}(e), \gamma^{\prime}\right\rangle}\left(\gamma^{\prime}<\gamma\right.$ and $\left.e<G_{0}\left(\gamma^{\prime}\right)\right)$, and

$$
f^{-1}(e) \cap p^{-1}[\eta] \subseteq Y \cap S_{\delta_{0}} .
$$

Note the strong use of the regularity of $Y$ and the fact that $G_{0} \mid \gamma$ is $\beta$-recursive.

LEMMA 3.3. For each $\gamma<\alpha$ :

(I) The set of permanent negative requirements of priority $<\gamma$ is $\beta$-finite and of $\beta$-cardinality less than $\kappa$.

(II) If $y$ belongs to a positive requirement of priority $\gamma$, but not to any permanent negative requirement of priority $<\gamma$, then $y \in X$.

Proof. By induction on $\gamma$. First the successor case. Fix $\gamma$ and assume that (I) and (II) hold for $\gamma$.

The verification of (I) for $\gamma+1$ begins with checking that the set of permanent negative requirements of priority $\gamma$ is $\beta$-recursive. Let $N$ be such a negative requirement. There are two ways in which $N$ can fail to be permanent. First, some element of $N$ is put in $X$. Second, $N$ is an $L$ - or $B$-requirement and is dropped because a similar requirement concerning the same reduction procedure and a lesser argument is destroyed. The similar requirement by virtue of its lesser argument is created before $N$ is. Let $\hat{N}$ be the set of all elements in requirements similar to $N$ and existing when $N$ is created. The bi-immunity of $X$ guarantees that all permanent negative requirements are of $\beta$-cardinality less than $\kappa$, so it is safe to restrict the search for the permanent to those smaller than $\kappa$.

Either way $N$ is temporary because an element is added to $X$ after being enumerated in $A$ or for the sake of a positive requirement of priority $\gamma^{\prime}<\gamma$. $A$ is $\beta$-recursive. By Lemma 3.2 , the set $P$ of all elements in positive requirements of priority $<\gamma$ is $\beta$-rec. Hence if $N$ is a $C$-requirement, $N$ is permanent iff $N \cap P=0$ and $N \cap A=0$. And if $N$ is an $L$ - or $B$-requirement, $N$ is permanent iff $(N \cup \hat{N}) \cap$ $P=0$ and $(N \cup \hat{N}) \cap A=0$.

Now the verification of (I) for $\gamma+1$ can be completed. Then the three types of negative requirements are considered in turn. Let $\sigma_{0}$ be a stage such that

$$
(\sigma)_{\sigma \geqslant \sigma_{0}}\left(\gamma^{\prime}\right)_{\gamma^{\prime} \leqslant \gamma+1}\left[G\left(\gamma^{\prime}, \sigma\right)=G_{0}\left(\gamma^{\prime}\right)\right] \text {, }
$$

and

$$
(e)_{e<G_{0}(\gamma+1)}\left[e \in \text { range } f \rightarrow e \in \text { range } f_{\sigma_{0}}\right] .
$$

By induction all $C$-requirements less than $\gamma$ satisfy (I). For each $e<G_{0}(\gamma)$, at most one permanent requirement $N_{\gamma, e}^{C}$ exists. Define $W$ to be

$$
\left\{e \mid e<G_{0}(\gamma) \&(E \sigma)_{\sigma \geqslant \sigma_{0}}\left(\text { a permanent } N_{\gamma, e}^{C} \text { is created at stage } \sigma\right)\right\} \text {. }
$$

Then $W \subseteq G_{0}(\gamma)<\beta^{*}$ is $\beta$-r.e., hence $\beta$-finite and of $\beta$-cardinality less than $\kappa$. There is a $\sigma_{1} \geqslant \sigma_{0}$ such that all permanent $N_{e, \gamma}^{C}$ 's associated with $W$ are created prior to stage $\sigma_{1}$. At stage $\sigma_{1}$ it is evident that the set of all such negative requirements has cardinality less than $\kappa$. 
As above, it is enough to consider $L$-requirements of priority $\gamma$. Let $U$ be

$$
\begin{aligned}
&\left\{e<G_{0}(\gamma) \mid\right.(E \sigma)_{\sigma \geqslant \sigma_{0}} \\
&\left.\left(e \text { is } \gamma \text {-inactive at stage } \sigma \text { because of some permanent } N_{\gamma, e, z}^{L}\right)\right\} .
\end{aligned}
$$

As above $U$ is $\beta$-finite. Thus there is a stage $\sigma_{2} \geqslant \sigma_{1}$ such that every permanent $N_{\gamma, e . z}^{L}$ created after stage $\sigma_{2}$ is associated with some computation $\left\{f_{\sigma}^{-1}(e)\right\}_{\sigma}^{X^{\sigma}}(z)$ that is correct (for $X$ ) and equal to $H(z)$. If there were $\kappa$ permanent $L$-requirements of priority $\gamma$, then $H$ would be $\beta$-recursive in 0 (an impossibility) as follows. It suffices by the $\beta$-bi-immunity of $H$ to show $\lambda \delta \mid H \uparrow \delta$ is $\beta$-recursive, where $\delta<\kappa$. Fix $\delta$ and go to some stage $\sigma_{3}$ beyond $\sigma_{2}$ where there is a permanent requirement $N_{\gamma, e, z}^{L}$ for each $z<\delta$ and some $e<G_{0}(\gamma)$. The existence of $\sigma_{3}$ is a consequence primarily of clause (i) of the definition of $L$-requirement. As each permanent $N_{\gamma, e, z}^{L}$ comes into being beyond stage $\sigma_{2}$, the associated $z$ increases monotonically towards $\kappa$ thanks to clause (i), and the manner in which $L$-requirements are created and destroyed.

That leaves only the $B$-requirements of priority $\gamma$. For each $e<G_{0}(\gamma)$, let $U_{e}$ be $\left\{z \mid\right.$ there is a permanent $\left.N_{\gamma, e, z}^{B}\right\} . U_{e}$ is an initial segment of $\beta^{*}$. If $\beta^{*}=\kappa$ or $\beta^{*}$ is singular, then $U_{e} \subseteq e$. In these two cases, $V=\left\{\langle e, z\rangle \mid z \in U_{e}\right\}$ is $\beta$-finite of $\beta$-cardinality less then $\kappa$, and so is the associated set of negative requirements.

If $\beta^{*}<{ }^{*} \kappa$ and regular, then $U_{e}$ is possibly an unbounded initial segment of $\beta^{*}$. The set $Q=\left\{e \mid e<G_{0}(\gamma) \& U_{e}=\beta^{*}\right\}$ is $\beta$-finite. Hence the set of permanent $B$-requirements $N_{\gamma, e, z}^{B}(e \in Q)$ is $\beta$-finite and of cardinality less then $\kappa$. For each $e \in G_{0}(\gamma)-Q, U_{e}$ is a proper initial segment of $\beta^{*}$. So by Proposition 2.4 , the set $\left\{\langle e, z\rangle \mid z \in U_{e} \& e \in G_{0}(\gamma)-Q\right\}$ has cardinality less than $\kappa$, and as well the associated set of permanent $B$-requirements.

To prove part (II) of Lemma 3.3, assume there is a positive requirement of priority $\gamma+1$ for $y$ created at stage $\sigma_{0}$. Assume $y \notin X$ with the intent of showing $y$ belongs to permanent negative requirement $\gamma^{\prime}$ for some $\gamma^{\prime}<\gamma+1$. Clearly $y$ belongs to some current negative requirement of higher priority at every stage $\sigma>\sigma_{0}$. Let $N$ be the set of all elements in any negative requirement of priority less than $\gamma+1$ and current at a stage past $\sigma_{0}$. If no element of $N$ lands in $X$, then all of $N$ is permanent and all is well since $y \in N$. Otherwise let $z$ be the least element of $N$ to be put in $X$, say at stage $\sigma_{1}>\sigma_{0}$. Then at stage $\sigma_{1}+1$, it must be that $y$ belongs to a negative requirement $N_{0}$ of priority less than $\gamma+1$ that was not injured by the addition of $z$ to $X$. But then $N_{0}$ must be permanent.

All that remains is the limit case. Let $\lambda<\alpha$ be a limit ordinal. The proof of (II) is the same as in the successor case. The proof of (I) is slightly modified. Define $\sigma_{0}$ as in the successor case. By induction: for each $\gamma<\lambda$, there is a $\tau \geqslant \sigma_{0}$ such that all permanent negative requirements of priority $\gamma$ are created by stage $\tau$. Let the least such $\tau$ by $\tau(\gamma)$. It follows from the induction hypothesis and (I) that $\tau(\gamma)$, as a function from $\lambda$ into $S_{\beta}$, is $\Sigma_{2}^{\beta}$. Since $\lambda$ is less than $\operatorname{tg} \operatorname{cf}(\beta), \tau(\gamma)$ is bounded by some $\sigma_{1} \geqslant \sigma_{0}$. As in the successor case, it follows that the set of all elements in permanent negative requirements of priority less than $\lambda$, and present at stage $\sigma_{1}$, has $\beta$-cardinality less than $\kappa$.

LemMA 3.4. $Y \leqslant_{\beta} X^{1 / 2}$. 
Proof. Since $Y$ is $\beta$-r.e., there is an $e_{0}$ such that $z \in Y \leftrightarrow\left\{e_{0}\right\}(z)$ converges. Thus

$$
F \subseteq \bar{Y} \leftrightarrow(z)_{z \in F}\left[\left\{e_{0}\right\}(z) \text { diverges }\right] \leftrightarrow\left\{e_{0}\right\} \times F \times \kappa \subseteq \overline{0^{1 / 2}} .
$$

Hence facts of the form $F \subseteq \bar{Y}$ can be $\beta$-recursively computed from $X^{1 / 2}$, since $0^{1 / 2} \leqslant{ }_{\beta} X^{1 / 2}$. The same must be checked for facts of the form $F \subseteq Y$. Let $e$ be $f(F)$, and $\gamma$ be the least $\rho$ such that $e<G_{0}(\rho)$. Then by Lemma 3.3, the union of all permanent negative requirements of priority less than $\gamma$ are bounded by some $\delta<\kappa$. Again by Lemma 3.3, all elements of $T_{\langle p(F), \gamma\rangle}-\delta$ which ever sit in a positive requirement are eventually put into $X$. That is,

$$
F \subseteq Y \leftrightarrow T_{\langle p(F), \gamma\rangle}-\delta \subseteq X
$$

More generally,

$$
F \subseteq Y \leftrightarrow(E \delta)_{\delta<\kappa}(E \gamma)_{\gamma<\alpha}\left[T_{\langle p(F), \gamma\rangle}-\delta \subseteq X\right] .
$$

The formula $[F, \gamma, \delta]$ is $\Pi_{1}^{X}$, hence $\beta$-recursive in $X$. Since $\delta$ and $\gamma$ are bounded by $\kappa$, it follows that $Y \leqslant_{\beta} X^{1 / 2}$.

LeMma 3.5. $X^{1 / 2} \leqslant_{\beta} Y$.

Proof. By Proposition 2.5(iii), it suffices to show $X^{\prime} \leqslant_{w \beta} Y$. First note that for each $\gamma<\alpha$, the set of positive requirements of priority less than or equal to $\gamma$ can be determined from $\beta$-finite information about $Y$, thanks to the regularity of $Y$. In more detail, let $\sigma_{0}$ be such that

$$
(\tau)_{\tau \geqslant \sigma_{0}}(e)_{e<G_{0}(\gamma)}\left[f^{-1}(e) \text { converges } \rightarrow f_{\tau}^{-1}(e) \text { converges }\right]
$$

and

$$
(\tau)_{\tau \geqslant \sigma_{0}}\left(\gamma^{\prime}\right)_{\gamma^{\prime} \leqslant \gamma}\left[G\left(\gamma^{\prime}, \tau\right)=G_{0}\left(\gamma^{\prime}\right)\right] .
$$

Since $0^{1 / 2} \leqslant_{\beta} Y, \sigma_{0}$ can be computed from $Y$ and $\gamma$. Let $\delta<\beta$ be such that for all $e<G_{0}(\gamma)$,

$$
e \in \text { range } f \rightarrow f^{-1}(e) \in S_{\delta} .
$$

$\delta$ exists by weak admissibility of $\beta$ and is computable from $Y$ since $0^{1 / 2} \leqslant_{\beta} Y$. $Y \cap S_{\delta}$ is $\beta$-finite because $Y$ is regular. $z$ belongs to a positive requirement of priority $\gamma$ iff for some $e^{\prime}$ and some $\gamma^{\prime} \leqslant \gamma, f\left(e^{\prime}\right)<G_{0}\left(\gamma^{\prime}\right) \& z$ is the $\eta$ th element of

$$
T_{\left\langle p\left(e^{\prime}\right), \gamma^{\prime}\right\rangle} \& p^{-1}[\eta] \cap e^{\prime} \subseteq Y \cap S_{\delta} .
$$

Next note that if $e \in X^{\prime}$, then eventually some permanent $C$-requirement $N_{\gamma, f(e)}^{C}$ is formed for $e$ as a consequence of the tameness of $X$. That is, if $e \in X^{\prime}$ then there exist unboundedly many $\sigma$ such that $\{e\}_{\sigma}^{X^{o}}(e)$ converges. At some such $\sigma, N_{\gamma, f(e)}^{C}$ is formed.

$e \in X^{\prime}$ is computed from $Y$ as follows. First $Y$ is used to determine $P$, the $\beta$-recursive set of positive requirements of priority $\leqslant \gamma$, where

$$
\gamma=\mu \gamma^{\prime}\left[f(e)<G_{0}\left(\gamma^{\prime}+1\right)\right] .
$$

Now for any $\lambda<\kappa$, let $P_{\lambda}$ denote those requirements in $P$ which are less than or equal to $\lambda$. (We are identifying here a positive requirement and the element it is trying to put into $X$.) Each $P_{\lambda}$ is $\beta$-finite with $\beta$-cardinality $<\kappa$. Thus for any $\lambda<\kappa$, 
$F_{\lambda}=P_{\lambda} \cap X$ is also $\beta$-finite. Note that $\left\{F_{\lambda} \mid \lambda<\kappa\right\}$ can be enumerated from $Y$ as $P$ can be computed from $Y$ and $X \leqslant_{\beta} 0^{1 / 2} \leqslant_{\beta} Y$. In addition, it follows from Lemma 3.3(I) that, for all sufficiently large $\lambda<\kappa, P \cap X=F_{\lambda} \cup P-P_{\lambda}$.

Then $e \in X^{\prime}$ iff

$$
(\exists \sigma)\left(\exists F_{\lambda}\right)\left(N_{\gamma, f(e)}^{C} \text { is current at stage } \sigma \& N_{\gamma, f(e)}^{C} \cap\left(F_{\lambda} \cup\left(P-P_{\lambda}\right)\right)=\varnothing\right) .
$$

This formula is $\Sigma_{1}^{S_{\beta}}$, hence weakly $\beta$-recursive in $Y$, since $0^{1 / 2} \leqslant_{\beta} Y$.

LEMMA 3.6. $X<{ }_{\beta} 0^{1 / 2}$.

Proof. $X \leqslant_{\beta} 0^{1 / 2}$ because $X$ is tamely $\beta$-r.e. Assume $H=\{e\}^{X}$. (Recall $H \equiv_{\beta} 0^{1 / 2}$.) Let $\gamma$ be the least $w$ such that $f(e)<G_{0}(w)$. Let $\sigma_{0}$ be such that $f_{\sigma_{0}}(e)$ converges and $(\tau)_{\tau \geqslant \sigma_{0}}\left(\gamma^{\prime}\right)_{\gamma^{\prime} \leqslant \gamma}\left[G\left(\gamma^{\prime}, \tau\right)=G_{0}\left(\gamma^{\prime}\right)\right]$. Define $z$ to be the least $y$ such that

$$
\left(e^{\prime}\right)\left[e^{\prime}<G_{0}(\gamma) \rightarrow \text { no permanent } N_{\gamma, e^{\prime}, y}^{L} \text { is created }\right] .
$$

According to Lemma 3.3, $z<\kappa$; in addition, there is a $\sigma_{1}>\sigma_{0}$ such that:

(1) for all $y<z$ there is a permanent $N_{\gamma, e^{\prime}, y}^{L}\left(e^{\prime}<G_{0}(\gamma)\right)$ created by stage $\sigma_{1}$, and

(2) every $\gamma$-inactive reduction procedure $e^{\prime}<G_{0}(\gamma)$ is seen to be inactive by stage $\sigma_{1}$.

Since the computation of $\{e\}_{\sigma}^{X^{o}}(z)$ is final and equal to $H(z)$ for all sufficiently large $\sigma$, it follows that a permanent $N_{\gamma, e^{\prime}, z}^{L}\left(e^{\prime}<G_{0}(\gamma)\right)$ is created, contrary to the definition of $z$.

LEMMA 3.7. $\kappa^{X}=\kappa$.

Proof. Suppose $\kappa^{X}<\kappa$. By Proposition 2.2, $\kappa^{X} \leqslant \beta^{*}$. And by Proposition 2.3, if $\beta^{*}$ is a singular $\beta$-cardinal, then $\kappa^{X}<\beta^{*}$. Choose $e$ so that $\{e\}^{X} \uparrow \kappa^{X}$ is unbounded in $\beta$. If $\kappa^{X}<\beta^{*}$, it may be assumed that $f(e)>\kappa^{X}$, and so that $B$-requirements are created in order to preserve $\{e\}^{X}$ on all $\kappa^{X}$.

Let $\gamma$ be such that $f(e)<G_{0}(\gamma)$. The reasoning of Lemma 3.3 shows there is a permanent $N_{\gamma, f(e), y}^{B}$ for each $y<\kappa^{X}$. Also there is a stage $\sigma$ by which all such requirements have been created. At that stage a bound on $\{e\}^{X} \uparrow \kappa^{X}$ is evident.

The proof of Theorem 3.1 is complete.

4. Applications of Theorem 3.1. First a degree-theoretic formulation of weak $\Sigma_{2}$ admissibility in the presence of weak $\Sigma_{1}$ inadmissibility. Then a result on $\alpha$-degrees above the complete $\alpha$-r.e. degree.

THEOREM 4.1. Suppose $\beta$ is weakly $\Sigma_{1}$ inadmissible. Then $\sigma 2 \operatorname{cf}(\beta) \geqslant \sigma 2 \mathrm{p}(\beta)$ iff there exists a $\beta$-recursively enumerable degree incomparable with $0^{1 / 2}$.

Proof. Suppose $\sigma 2 \operatorname{cf}(\beta)<\sigma 2 \mathrm{p}(\beta)$ and $B$ is a $\beta$-r.e. set incomparable with $0^{1 / 2}$. By Proposition 2.9, $B$ can be assumed to be regular. But then the effective disjoint union of $B$ and a regular, $\beta$-r.e. representative of $0^{1 / 2}$ is regular and of higher degree than $0^{1 / 2}$, an impossibility according to 2.9 .

Suppose $\sigma 2 \operatorname{cf}(\beta) \geqslant \sigma 2 \mathrm{p}(\beta)$. By 2.9 there exists a regular, complete $\beta$-r.e. set $C$. Theorem 3.1 provides a bi-immune, $\beta$-r.e. set $X \subseteq \kappa$ such that $X<{ }_{\beta} 0^{1 / 2}, X^{1 / 2} \equiv{ }_{\beta} C$ and $\kappa^{X}=\kappa$. Let $\mathcal{Q}$ be the admissible collapse of $S_{\beta}$ (as in $\S 2$ ), and let $\hat{\mathcal{Q}}$ be $\langle\mathcal{Q}, X\rangle$. Since $\kappa^{X}=\kappa, \hat{\mathbb{Q}}$ is an admissible structure. The proof of Shore's splitting theorem [9] 
for $\alpha$-recursion theory is valid in $\hat{\mathcal{Q}}$. His argument is dynamic, that is, it is based entirely on simultaneous enumerations, and so makes no use of collapsing arguments legal in $L(\alpha)$ but banned in $\langle L(\alpha), Z\rangle$ for most $Z$ 's. Thus there is an $\hat{Q}$-r.e. bi-immune $Y \subseteq \kappa$ such that $Y \$ \hat{\hat{e}} 0^{1 / 2}$ and $0^{1 / 2} \$ \hat{\mathfrak{e}} Y$. The bi-immunity of $Y$ implies $Y$ is $\beta$-incomparable with $0^{1 / 2}$. By $2.9 Y$ has a $\beta$-r.e. representative.

The second application of Theoremm 3.1 is, to be precise, an application of a relativization of 3.1. A great deal of the thinking in $\$ 2$ has been relativized by Maass $[3,6]$, and the results needed here can be sketched as follows. Assume $\alpha$ is $\Sigma_{1}$ admissible and weakly $\Sigma_{2}$ inadmissible (i.e. $\alpha>\sigma 2 \operatorname{cf}(\alpha) \geqslant \sigma 2 \mathrm{p}(\alpha)$ ). Let $C$ be a complete, regular $\Sigma_{1}^{\alpha}$ set. Define $S$ by $\left\langle S_{\alpha}, \varepsilon, C\right\rangle$. Then $\Sigma_{1}^{S}=\Sigma_{2}^{\alpha}$, and tamely $\Sigma_{1}^{S}=$ tamely $\Sigma_{2}^{\alpha}$. ( $B$ is tamely $\Sigma_{2}^{\alpha}$ if $\left\{K \mid K \in S_{\alpha} \& K \subseteq B\right\}$ is $\Sigma_{2}^{\alpha}$.) Let $f: \sigma 2 \operatorname{cf}(\alpha) \rightarrow \alpha$ be $\Sigma_{2}^{\alpha}$ and have unbounded range. The half-jump of $X$ in the sense of $S$ should be $\left\{\langle e, z, \sigma\rangle \mid\{e\}^{X}(z)\right.$ converges by stage $\left.f(\sigma)\right\}$, and it is if $X$ is a tamely $\Sigma_{2}^{\alpha}$ set such that $C \leqslant_{\alpha} X$. Let $Z$ denote a hyperregular $\alpha$-r.e. set. Then $Z^{\prime}$, the $\alpha$-jump of Shore [10], is a tamely $\Sigma_{2}^{\alpha}$ set. It is thus reasonable, following Maass [11], to define $Z^{3 / 2}$ to be the half-jump (in the sense of $S$ ) of $Z^{\prime}$. Maass [11] showed (for weakly $\Sigma_{2}$ inadmissible $\alpha$ among others) that $0^{3 / 2}$ is the greatest $\alpha$-degree with a tamely $\Sigma_{2}^{\alpha}$ representative.

THEOREM 4.2. Suppose $\alpha$ is $\Sigma_{1}$ admissible and weakly $\Sigma_{2}$ inadmissible. If $Y$ is $\Sigma_{2}^{\alpha}$, regular and $0^{3 / 2} \leqslant_{\alpha} Y$, then there exists an incomplete, hyperregular $\alpha-r . e . Z$ such that $Z^{3 / 2} \equiv{ }_{\alpha} Y$.

Proof. The structure $S\left(=\left\langle S_{\alpha}, \varepsilon, C\right\rangle\right)$ is by supposition weakly $\Sigma_{1}$ admissible. It was noted that 3.1 had a "hidden assumption", the weak $\Sigma_{1}$ admissibility of $\beta$ over $0^{1 / 2}$. The corresponding assumption for $S$ is $\sigma 3 \operatorname{cf}(\alpha) \geqslant \sigma 3 \mathrm{p}(\alpha)$, and it holds if there exists a regular, $\Sigma_{2}^{\alpha}, Y>_{\alpha} 0^{3 / 2}$. The proof of 3.1 is valid for $S$, and so there exists a tame $\Sigma_{2}^{\alpha} X$ such that $0^{\prime} \leqslant_{\alpha} X$ and the half-jump (in the sense of $S$ ) of $X$ has the same $\alpha$-degree as $Y$. According to Maass [11], $X \equiv_{\alpha} Z^{\prime}$ for some incomplete, hyperregular $\alpha$-r.e. $Z$.

5. A density theorem. In a moment an indirect proof will be given of the density of the $\beta$-r.e. degrees when $\beta$ is weakly $\Sigma_{1}$ admissible and weakly $\Sigma_{2}$ admissible. A direct proof could be given by adapting the density argument of Shore [12] for the $\Sigma_{1}$ admissible case. But that would be much longer than what follows. The entire matter is greatly simplified by Maass's work on the admissible collapse, in particular Proposition 2.9.

Suppose $\beta$ satisfies the above assumptions. Let $b$ be a tamely $\beta$-r.e. degree. Choose $B \in b$ to be $\beta$-immune and $\mathcal{Q}$-r.e., where $\mathcal{Q}$ is the admissible collapse of $S_{\beta}$. Clearly $B$ is tamely $\beta$-r.e. Define $\mathbb{Q}_{b}$ to be $\langle Q, B\rangle$ if $\langle Q, B\rangle$ is admissible, and the admissible collapse of $\langle Q, B\rangle$ otherwise.

By Proposition 2.9 every $\beta$-r.e. degree has a $\beta$-bi-immune representative $A \subseteq \kappa$ that is $Q_{b}$-r.e. for some tamely $\beta$-r.e. degree $b \leqslant_{\beta} A$. Thus each $\beta$-r.e. degree corresponds to some $\mathbb{Q}_{b}$-r.e. degree in at least one $\mathbb{Q}_{b}$. The converse also holds by 2.9. In addition the relation $\leqslant_{\mathbb{Q}_{b}}$ for $\mathbb{Q}_{b}$-r.e. degrees corresponds to the relation $\leqslant_{\beta}$ for $\beta$-r.e. degrees. Thus the $\beta$-r.e. degrees can be studied by studying the $\mathbb{Q}_{b}$-r.e. degrees. 
Maass observed that the splitting theorem (for degrees) holds for $S_{\beta}$ because Shore's proof of splitting for an admissible ordinal holds in every $\mathcal{Q}_{b}$.

Theorem 5.1. Suppose $\beta$ is weakly $\Sigma_{1}$ admissible over 0 and $0^{1 / 2}$. Let $A$ and $C$ be $\beta$-r.e. sets such that $A<{ }_{\beta} C$ and $C$ is regular. Then there exists a regular, $\beta$-r.e. set $B$ such that $A<{ }_{\beta} B<{ }_{\beta} C$.

Proof. By $2.9 C$ is tamely $\left\langle Q, C_{0}\right\rangle$-r.e. for some tamely $\beta$-r.e. $C_{0}$, and the same holds for $A$ relative to some tamely $\beta$-r.e. $A_{0}$. Let $c_{0}$ be the $\beta$-degree of $C_{0}$, and $a_{0}$ that of $A_{0}$.

Shore's density theorem [12] holds in $\mathbb{Q}_{b}$ for every tamely $\beta$-r.e. degree $b$. This is not hard to verify, but does need to be checked, because Shore's proof makes use of a collapsing argument not valid in all admissible structures of the form $\left\langle L_{\kappa}, W\right\rangle$. All goes well in $Q_{b}$ because of the tamely r.e. nature of $b$. See Homer [13] for details.

Case 1. $A \oplus C_{0} \equiv_{\beta} C$. It follows that $C$ is tamely $\beta$-r.e. over $A_{0}$. Thus the degrees of $C$ and $A$ both lie in the structure $\mathbb{Q}_{a_{0}}$, and the density theorem for $\mathbb{Q}_{a_{0}}$ yields the desired $B$.

Case 2. $A \oplus C_{0}<_{\beta} C$.

Subcase 2.1. $A<_{\beta} A \oplus C_{0}$. Let $B$ be $A \oplus C_{0}$, more precisely a regular, $\beta$-r.e. set equivalent to $A \oplus C_{0}$.

Subcase 2.2. $A \equiv_{\beta} A \oplus C_{0}$. Let $B_{0}=A_{0} \oplus C_{0}$. Then $B_{0} \leqslant_{\beta} A, B_{0}$ is tamely $\beta$-r.e., and the degrees of $C$ and $A$ both lie in the structure $Q_{b_{0}}$. Finish as in Case 1.

6. Open questions. It is far from clear what roles regularity and weak $\Sigma_{2}$ admissibility play in Theorems 3.1 and 5.1. It is possible that the following variation on 3.1 is provable: Suppose $Y$ is $\beta$-r.e. and $0^{1 / 2} \leqslant_{\beta} Y$; then there exists a $\beta$-r.e. $X$ such that $X<_{\beta} 0^{1 / 2}$ and $X^{1 / 2} \equiv_{\beta} Y$. Very little is known about r.e. set constructions in which the advantages of regularity are not exploited. It would be pleasing if the $\beta$-r.e. degrees were dense for every weakly $\Sigma_{1}$ admissible ordinal, but it is difficult to see how to begin in the face of strong $\Sigma_{2}$ inadmissibility. It is tempting to try to reduce the problem to the regular $\beta$-r.e. degrees below $0^{1 / 2}$, and to construe the desired intermediate $\beta$-r.e. degree as the half-jump of one below.

\section{BIBLIOGRAPHY}

1. R. M. Friedberg, A criterion for completeness of degrees of unsolvability, J. Symbolic Logic 22 (1957), 159-160.

2. G. E. Sacks, Recursive enumerability and the jump operator, Trans. Amer. Math. Soc. 108 (1963), 223-239.

3. W. Maass, Inadmissibility, tame r.e. sets and the admissible collapse, Ann. Math. Logic 13 (1978), 149-170.

4. S. D. Friedman and G. E. Sacks, Inadmissible recursion theory, Bull. Amer. Math. Soc. 83 (1977), 255-256.

5. S. D. Friedman, Recursion on inadmissibile ordinals, Ph.D. Thesis, M.I.T., 1976.

6. W. Maass, On $\alpha$ - and $\beta$-recursively enumerable degrees, Ann. Math. Logic 16 (1979), 205-231.

7. G. E. Sacks and S. G. Simpson,-The $\alpha$-finite injury method, Ann. Math. Logic 4 (1972), 323-367.

8. S. G. Simpson, Degree theory on admissible ordinals, Generalized Recursion Theory, North-Holland, Amsterdam, 1974, pp. 165-193.

9. R. A. Shore, Splitting on $\alpha-r . e$. set, Trans. Amer. Math. Soc. 204 (1975), 65-77.

10. $351-363$ , On the jump of an $\alpha$-recursively enumerable set, Trans. Amer. Math. Soc. 217 (1976), 
11. W. Maass, Contributions to $\alpha$ - and $\beta$-recursion theory, Habilitationsschrift, Ludwig-MaximilianasUniversität Munchen, 1977.

12. R. A. Shore, The $\alpha$-r.e. degrees are dense, Ann. Math. Logic 9 (1976), 123-155.

13. S. E. Homer, Priority arguments in $\beta$-recursion theory, Ph.D. Thesis, M.I.T., 1978.

Department of Mathematics, De Paul University, Chicago, Illinois 60614

Department of Mathematics, Harvard University, Cambridge, Massachusetts 02138 (current address)

Department of Mathematics, Massachusetts Institute of Technology, Cambridge, MasSACHUSETTS 02139

Current address (S. Homer): Department of Mathematics, Boston University, Boston, Massachusetts 02215 